Kenneth M. Weiss

Department of Anthropology, University of Michigan, Ann Arbor, Michigan 48104, U.S.A.

Received 18 August 1971

\section{A Generalized Model for Competition between Hominid Populations}

\begin{abstract}
A simple mathematical model can represent competition between two hominid populations which includes elements of both trophic preemption and killing. The ultimate outcome of such competition can be determined if certain parameters are estimated. Various values of these parameters can represent various types of competition which occur between hominid groups. The model is used to argue that the two-species explanation of Australopithecine fossils is unrealistic and that a one-species view is more compatible with the facts. This model lends itself to first-order approximation of many situations of interest to anthropologists. The form of the equations for sevcral situations is given, as is a solution for the ultimate equilibrium population densities of the two competing groups.
\end{abstract}

Mathematical models for competition between two animal populations have generally dealt heretofore either with predation of one on the other or with indirect trophic competition between them; these are discussed in Slobodkin (1961) or Watt (1968). The various forms of such models do not consider a more generalized competition including elements of both direct and indirect competition. This is necessary to analyze interactions between competing hominid or human groups, for they compete both trophically and physically. A simple generalized model will be shown here, and followed by an example illustrating its use in problems of human ecology and evolution.

By combining aspects of existing models, we can form equations for generalized competition as

where:

$$
\begin{aligned}
\frac{\mathrm{d} N_{1}}{\mathrm{~d} t} & =\frac{r_{1} N_{1}\left(K_{1}-N_{1}-a N_{2}\right)}{K_{1}}-C N_{1} N_{2} \\
\frac{\mathrm{d} N_{2}}{\mathrm{~d} t} & =\frac{r_{2} N_{2}\left(K_{2}-N_{2}-b N_{1}\right)}{K_{2}}-D N_{1} N_{2}
\end{aligned}
$$

$N_{1}, N_{2}=$ actual population densities,

$K_{1}, K_{2}=$ maximum population densities in absence of competitors (carrying capacity),

$r_{1}, r_{2}=$ population growth rates,

$b, a=$ per capita trophic pre-empting competition coefficient,

$D, C=$ per capita killing competition coefficient.

All values except the $N$ 's are constants (long-term averages). On the right side of these equations the first term represents the intrinsic biological rate of increase of the population in isolation minus the self-damping factor which slows growth as carrying capacity is neared and the damping factor due to the trophic pre-emption of its members by members of the other population. The second term is the loss of members due to their being killed by the other group, or the gain in members due to the food gained by eating prey from the competing population.

These equations use the simplest and most tractible form of the killing term. Other forms have been suggested, but the mathematical complexity thus introduced is not 
worth the small extra information made available when analyzing the very general problems of central interest to anthropologists. A discussion of the more complex forms of the killing term may be found in Watt (1968) or see Keyfitz (1968).

To determine the outcome of competition following the form of the model suggested here, one must analyze the isoclines for each population (those values of $N_{1}$ and $N_{2}$ for which each population does not change) given by $\mathrm{d} N_{1} / \mathrm{d} t=0$ and $\mathrm{d} N_{2} / d t=0$. These isoclines are linear, and therefore the result of competition can be determined by the relative values of the $N_{1}$ and $N_{2}$ intercepts. This procedure is outlined by Slobodkin (1961). We need to evaluate the relative magnitudes of

and

$$
\frac{r_{1} K_{1}}{r_{1} a+C K_{1}} \text { compared to } K_{2}
$$

$$
\frac{r_{2} K_{2}}{r_{2} b+D K_{2}} \text { compared to } K_{1} \text {. }
$$

There are four possible outcomes. These are shown in Table 1 along with the ultimate

Table 1

\begin{tabular}{|c|c|c|c|}
\hline Case No. & If & And if & Result of the competition will be* \\
\hline & & & Stable coexistence at \\
\hline (1) & $\frac{r_{1} K_{1}}{r_{1} a+C K_{1}}>K_{2}$ & $\frac{r_{2} K_{2}}{r_{2} b+D K_{2}}>K_{1}$ & $\begin{array}{l}N_{1}=\frac{r_{2}\left[K_{2}\left(r_{1} a+C K_{1}\right)-r_{1} K_{1}\right]}{\left(r_{1} a+C K_{1}\right)\left(r_{2} b+D K_{2}\right)-r_{1} r_{2}} \\
N_{2}=\frac{r_{1}\left[K_{1}\left(r_{2} b+D K_{2}\right)-r_{2} K_{2}\right]}{\left(r_{1} a+C K_{1}\right)\left(r_{2} b+D K_{2}\right)-r_{1} r_{2}}\end{array}$ \\
\hline (2) & $\frac{r_{1} K_{1}}{r_{1} a+C K_{1}}>K_{2}$ & $\frac{r_{2} K_{2}}{r_{2} b+D K_{2}}<K_{1}$ & $\begin{array}{l}\text { Only } N_{1} \text { survives: } \\
N_{1}=K_{1} \\
N_{2}=0\end{array}$ \\
\hline (3) & $\frac{r_{1} K_{1}}{r_{1} a+C K_{1}}<K_{2}$ & $\frac{r_{2} K_{2}}{r_{2} b+D K_{2}}>K_{1}$ & $\begin{array}{l}\text { Only } N_{\mathrm{a}} \text { survives: } \\
N_{1}=0 \\
N_{\mathrm{a}}=K_{\mathrm{a}}\end{array}$ \\
\hline (4) & $\frac{r_{1} K_{3}}{r_{1} a+C K_{1}}<K_{2}$ & $\frac{r_{2} K_{2}}{r_{2} b+D K_{2}}<K_{1}$ & $\begin{array}{l}\text { Unstable at same values as Case 1; eventual } \\
\text { elimination of one species, depending on the } \\
\text { initial concentrations (result in either Case } 2 \text { or } \\
\text { Case 3). }\end{array}$ \\
\hline
\end{tabular}

Outcome of competition

* The Gase 1 and Gase 4 results will always be mathematically defin ed under the given conditions.

equilibrium population sizes and the stability of that equilibrium. Since these are expressed in terms of the constants in the original equations, solutions to actual problems will rest on evaluating these constants.

Regardless of the outcome of competition, there are, for specific kinds of population interactions, general forms which can be predicted for several of the constants in the equations $[(1),(2)]$. A few of these specific types of interactions are:

\section{(1) Pure trophic competition}

If both groups compete only by eating each other's potential food supply, then there will be no killing term and the values of $C$ and $D$ will be zero (0). The equations then reduce to the standard Gaussian competition equations. 


\section{(2) Pure predation}

If $N_{2}$ preys on $N_{1}$ and derives added food thereby, then we can interpret $C$ as the perpredator predation rate. The value of $D$ will be a measure of the rate at which $N_{2}$ increases for each prey captured. If there are $C N_{1} N_{2}$ prey captured, and they are converted to members of group $N_{2}$ at a rate, $g$, then $D$ will be a negative loss equal to the product of $g$ and the prey; that is, $D=-g C$. In a case of pure predation, there will be no trophic competition, and so $a$ and $b$ will be zero $(0)$. The resulting model differs from the classic Lotka-Volterra system in that it puts a limit on the sustaining ability of the environment for both $N_{1}$ and $N_{2}$, and also that it expresses the fact that omnivores, even when acting as predators, derive food from more than just their prey. The omnivore's population will thus be regulated by more than just the supply of prey.

\section{(3) Warfare}

In a case of steady killing of members of both groups by members of the other, but without cannibalism, the values of $C$ and $D$ represent the "combat efficiencies" of each member of each group. Both will be positive constants since the killing detracts from both populations. The trophic competition will be absent, so $a$ and $b$ will be zero (0). If there is trophic competition along with warfare, the original equations $[(1),(2)]$ express the form the relationship will take.

Table 2 summarizes these and other types of competition between two hominid groups and the general form the constants will take. The equations can obviously be modified for variants of these situations. For example, in every case shown above, a "rarefaction"term might be included if both species or groups were exposed to a non-selective hazard imposed from without (such as another predator). This could be done by inserting a coefficient of $\left(1-x / r_{1}\right)$ in the first and $\left(1-x / r_{2}\right)$ in the second equations before the first

Table 2

Form of competition equations for some specific situations

\begin{tabular}{llc}
\hline \multicolumn{1}{c}{ Situation } & $\mathrm{d} N_{1} / \mathrm{d} t=$ & $\mathrm{d} N_{2} / \mathrm{d} t=$ \\
\hline Pure trophic competition & $r_{1} N_{1} \frac{\left(K_{1}-N_{1}-a N_{2}\right)}{K_{1}}$ & $r_{2} N_{2} \frac{\left(K_{2}-N_{2}-b N_{1}\right)}{K_{2}}$ \\
Pure predation of $N_{2}$ on $N_{1}$ & $r_{1} N_{1} \frac{\left(K_{1}-N_{2}\right)}{K_{1}}-C N_{2} N_{2}$ & $r_{2} N_{2} \frac{\left(K_{2}-N_{2}\right)}{K_{2}}+g C N_{1} N_{2}$ \\
One way attacks of $N_{2}$ on $N_{1}$ & $r_{1} N_{1} \frac{\left(K_{1}-N_{1}\right)}{K_{1}}-C N_{1} N_{2}$ & $r_{2} N_{2} \frac{\left(K_{2}-N_{2}\right)}{K_{2}}$ \\
Steady warfare & $r_{1} N_{1} \frac{\left(K_{1}-N_{1}\right)}{K_{1}}-C N_{1} N_{2}$ & $r_{2} N_{2} \frac{\left(K_{2}-N_{2}\right)}{K_{2}}-D N_{1} N_{2}$ \\
$\begin{array}{l}\text { Mixed trophic and predation } \\
\text { Competition }\end{array}$ & $r_{1} N_{1} \frac{\left(K_{1}-N_{1}-a N_{2}\right)}{K_{1}}-C N_{1} N_{2}$ & $r_{2} N_{2} \frac{\left(K_{2}-N_{2}-b N_{1}\right)}{K_{2}}+g C N_{1} N_{2}$ \\
$\begin{array}{l}\text { Mixed trophic and warfare } \\
\text { Competition }\end{array}$ & $r_{1} N_{1} \frac{\left(K_{1}-N_{1}-a N_{2}\right)}{K_{1}}-C N_{1} N_{2}$ & $r_{2} N_{2} \frac{\left(K_{2}-N_{2}-b N_{1}\right)}{K_{2}}-D N_{1} N_{2}$ \\
$\begin{array}{l}\text { Mixed trophic and warfare } \\
\text { Competition with "rarefaction" }\end{array}$ & $r_{1} N_{1} \frac{\left(\left(1-x / r_{1}\right) K_{1}-N_{1}-a N_{2}\right)}{K_{1}}$ & $r_{2} N_{2} \frac{\left(\left(1-x / r_{2}\right) K_{2}-N_{2}-b N_{1}\right)}{K_{2}}$ \\
& $-C N_{1} N_{2}$ & $-D N_{1} N_{2}$ \\
\hline
\end{tabular}


term in the parcntheses. The rarefaction factor, $x$, is the death rate from the hazard. The form for the competition analysis in this case is shown in the Appendix.

The equations of competition can now be used to analyze specific problems. Following is one such case in the analysis of hominid evolution.

\section{Example: the Number of Early Hominid Species}

There is considerable disagreement as to whether the fossil record from the early Pleistocene provides evidence for two lines of evolution, Australopithecus africanus and Paranthropus robustus and their descendents, or whether it shows only one line, Homo africanus. The common view that there were two also holds that they were sympatric over millions of years and over a wide geographical area including most of the tropical Old World (Robinson, 1967). The africanus line is said to have persisted and the robustus line to have become extinct. Opinion varies as to the extent to which the two competed, but surely there must have been some trophic competition and combat is often considered to have occurred also. Some would see predation of africanus on robustus as well.

Robustus is thought to have been strictly herbivorous and africanus omnivorous. Masticatory anatomy is used to discriminate these dietary adaptations (Robinson, 1963), and Schaffer (1968) has discussed these differences in terms of character displacement which, he feels, has made strictly sympatric finds more different from each other than finds of one species only. Even with dietary specializations, however, one can expect trophic competition at least to the extent that the africanus could have eaten some of the plants edible by robustus, and the latter would have been as able as chimpanzees and baboons to scavenge occasional meat kills which would also be available to africanus.

Could competition between such long-term sympatric populations explain the extinction of one of them? $\dot{W}$ can look at this extinction from the point of view of equations $[(1),(2)]$. If robustus is the population represented by $N_{1}$, then from Table 1 , the conditions for extinction are

and

$$
\frac{r_{1} K_{1}}{r_{1} a+C K_{1}}<K_{2}
$$

$$
\text { or, from (5) and (6), } \quad \begin{gathered}
\frac{r_{2} K_{2}}{r_{2} b+D K_{2}}>K_{1} \\
\frac{\left(r_{1} a+C K_{1}\right) / r_{1}}{\left(r_{2} b+D K_{2}\right) / r_{2}}>\left(\frac{K_{1}}{K_{2}}\right)^{2} .
\end{gathered}
$$

The numerator and denominator on the lcft might be called the total competitive effect (TCE) of population $N_{2}$ on $N_{1}$ in the first case and $N_{1}$ on $N_{2}$ in the second. The outcome of the competition is thus dependent on the ratio of the TCE values to that of the carrying capacities for the two species squared. To evaluate whether extinction is reasonable one must therefore estimate the likely carrying capacities for the early hominids.

The highest density that early hominid omnivores would be expected to have attained would presumably be close to that of modern hunter-gatherers who have been so adapted for millions of years of evolution. Their densities are on the order of 0.1 per square mile. Primate herbivores, on the other hand, in areas similar to those inhabited by early hominids range in density from about $2 / 3$ per square mile for the patas monkey to over 13 per square mile for some chimpanzees and baboons. Most are well over 1 per square mile. 
Thus we can be conservative and estimate that the herbivore densities were about a factor of 10 greater than those for the omnivore: the omnivorous niche allows specialization on another food source, but costs a factor of 10 in density. That is, we have $K_{1}=10 K_{2}$.

With this density estimate, the right side of equation (7) becomes

$$
\left(\frac{K_{1}}{K_{2}}\right)^{2}=\left(\frac{10 K_{2}}{K_{2}}\right)^{2}=-100,
$$

and thus, that for the alleged extinction to have occurred the TCE of the omnivore would have had to have become more than 100 times that of the herbivore. Evaluating the specific constants in (7) is difficult when warfare or predation are included, but in the particular case where there is no such competition, we have, from (7) and (8),

$$
a>100 b \text {. }
$$

Such exaggerated trophic competition between similar animals is unlikely to say the least. And even with killing, the competitive disparity still must be over 100 to 1 between the two.

Both forms are assumed to have lived stably and sympatrically over a wide area and for a long time, both had similar brains, both were anatomically upright, bipedal, and hand-using, and both had a rudimentary culture. It is therefore difficult to accept a competitive disparity of over 100 to 1 between two species with such similar ecologies and morphologies. What could have caused it? It cannot be argued that the stable condition which persisted for millions of years found a nearly 100 to 1 disparity and that the extinction represented merely a slight advancement of africanus over robustus, for were that the case then for the long time in which they were coexisting the africanus was badly exploiting robustus, and in such a case the marked anatomical specializations would not have been called for. If africanus was taking that much food from robustus, he would not need strong adaptations for meat-eating.

The explanation cannot rest heavily on predation either. It is taken as axiomatic by biologists that predators do not exterminate their prey. The competitive ability of the United States against North Vietnam in a very uneven war is far from 100 to 1 . Human groups have rarely, if ever, completely exterminated other human groups through warfare, and certainly never on the scale that would have been required here.

Hence the two-species view of early hominid evolution is not compatible with this model of population ecology, for it fails to give sufficient evidence to explain the extinction of robustus. On the other hand, dental and anatomical evidence discussed by Brace (1971) and Wolpoff $(1970,1971)$ seems to support a simpler single-species view. This is more reasonable from the point of view of population ecology.

\section{Conclusion}

The equations for generalized competition between hominid groups are simple ones and easy to apply if certain real parameters can be estimated. While they are approximate and would fail to give an accurate description of the expectable approaches to equilibrium, they can give general results and a figure for the eventual sizes of the two competing populations. This can be of use in many cases of interaction of interest to anthropologists. Cases which come to mind include the Neanderthal extinction problem, relations between competing tribal or linguistic populations, and colony-colonizer relationships.

With modification the equations could be applied to situations as diverse as restricted warfare between nations (as in medieval Europe), all-out war, resource exploitation of one 
population by another, and an estimate of the relative competitive merits of juxtaposed different cultural forms. Eventually it should be possible to develop $N$ differential equations for competition between $N$ populations (nations), and with the aid of computer techniques to solve for equilibrium densities and so forth. Finally, more complex models (e.g. for the killing term) might be compared to the simple one to determine the usefulness of the finer approximations; with the use of computers such models might be worth the effort required to solve the equations.

The assistance of Professors F. B. Livingstone and C. L. Brace during the development of this paper is gratefully acknowledged.

\section{References}

Brace, C. I. (1971). Sexual dimorphism and the Australopithecine identity crisis. Paper given at the annual meeting of the American Association of Physical Anthropologists, Boston.

Keyfitz, N. (1968). Introduction to the Mathematics of Population. London: Addison-Wesley.

Robinson, J. T. (1963). Adaptive radiation in the Australopithecines and the origin of man. In (F. C. Howell \& F. Bourliere, Eds) African Ecology and Human Evolution, pp. 385-416. Chicago: Aldine.

Robinson, J. T. (1967). Variation and the taxonomy of early hominids. In (T. Dobzhansky, M. Hecht, \& W. Steere, Eds), Evolutionary Biology, pp. 69-100. New York: Appleton-Century-Crofts.

Schaffer, W. M. (1968). Character displacement and the evolution of the Hominidae. American Naturalist 102, 559-571.

Slobodkin, L. B. (1961). Growth and Regulation of Animal Populations. New York: Holt, Rinehart, Winston. Watt, K. E. F. (1968). Ecology and Resource Management. New York: McGraw-Hill.

Wolpoff, M. H. (1970). The evidence for multiple hominid taxa at Swartkrans. American Anthropologist 72, 576-607.

Wolpoff, M. H. (1971). Metric trends in hominid dental evolution. Gase Western Reserve University Studies in Anthropology, 2. Press of Case Western Reserve Univ., Cleveland.

\section{Appendix}

With rarefraction at a rate ' $x$ ' on both populations, the equations (3) and (4) would be:

$$
\begin{aligned}
& \frac{\left(r_{1}-x\right) K_{1}}{r_{1} a+C K_{1}} \text { compared to }\left(1-x / r_{2}\right) K_{2} \\
& \frac{\left(r_{2}-x\right) K_{2}}{r_{2} b+D K_{2}} \text { compared to }\left(1-x / r_{1}\right) K_{1} .
\end{aligned}
$$

These values would replace those in Table 1 to establish the four cases.

The resulting equilibrium values for $N_{1}$ and $N_{2}$ would be as follows:

Case 1 and Case 4: $\quad N_{1}=\frac{\left(r_{2}-x\right) K_{2}\left(r_{1} a+C K_{1}\right)-r_{2}\left(r_{1}-x\right) K_{1}}{\left(r_{1} a+C K_{1}\right)\left(r_{2} b+D K_{2}\right)-r_{1} r_{2}}$

$$
N_{2}=\frac{\left(r_{1}-x\right) K_{1}\left(r_{2} b+D K_{2}\right)-r_{1}\left(r_{2}-x\right) K_{2}}{\left(r_{1} a+C K_{1}\right)\left(r_{2} b+D K_{2}\right)-r_{1} r_{2}}
$$

Case 2: $\quad N_{1}=\left(1-x / r_{1}\right) K_{1}$

$N_{2}=0$

Case 3: $\quad N_{1}=0$

$$
N_{2}=\left(1-x / r_{2}\right) K_{2}
$$

